\title{
Intimate Partner Violence Among Transgender Youth: Associations with Intrapersonal and Structural Factors
}

\author{
Tamar Goldenberg, MPH, Laura Jadwin-Cakmak, MPH, and Gary W. Harper, PhD, MPH
}

\begin{abstract}
Intimate partner violence (IPV) is a serious public health problem, linked with a number of poor health outcomes. Some research demonstrates that transgender and gender nonconforming (TGNC) youth may experience IPV at similar rates to heterosexual cisgender women; however, there is a dearth of data examining the experiences of IPV among this population. The exclusion of TGNC youth in IPV research and public health interventions is problematic because it contributes to a traditional gender-based heterosexual model of IPV that ignores the specific needs of TGNC populations. Given the pervasiveness of transphobic stigma in the United States and the complexities of the lives of TGNC youth, it is important to understand how both structural and individual factors are associated with IPV among TGNC youth. Using data from a cross-sectional survey of 131 TGNC youth from 14 different cities in the United States representing various regions of the country, this study explores the associations between a range of structural and intrapersonal factors and experiences of IPV victimization among TGNC youth. Separate logistic regression models were fit to examine the relationships between structural and intrapersonal factors and IPV. Structural results found that TGNC youth experiencing extreme victimization, a history of incarceration, and participation in sex work were much more likely to report IPV. The only intrapersonal variable that was associated with IPV was depressive symptomatology; however, this relationship was strong, with participants being nearly eight times more likely to report IPV if they experienced symptoms of depression. Gaining a better understanding of the complexity of both structural and intrapersonal factors among TGNC youth will greatly inform the development of IPV prevention and services for TGNC youth experiencing this type of violence.
\end{abstract}

Keywords: intimate partner violence, transgender, youth, structural factors intrapersonal factors

\section{Introduction}

$\mathbf{T}$ RANSPHOBIC STIGMA IN the United States contributes to violence targeted at transgender and gender nonconforming (TGNC) adolescents and young adults (i.e., individuals whose gender identity differs from their sex assigned at birth or whose gender identity does not fall into conventional binary categories of male or female, hereafter referred to as "TGNC youth"). Often, this violence takes the form of transphobic victimization, which can occur as sexual, physical, and verbal violence and harassment that occurs because someone is presumed to be TGNC (Lombardi et al. 2002; Stotzer 2009).

Another less-commonly researched form of violence experienced by TGNC individuals is intimate partner violence (IPV) (Dank et al. 2014; James et al. 2016; LangenderferMagruder et al. 2016). According to the World Health Organization (2010), IPV refers to "any behavior within an intimate relationship that causes physical or psychological harm, including acts of physical aggression, sexual coercion, psychological abuse, and controlling behaviors." Most IPV research has focused on IPV perpetrated against cisgender women (i.e., individuals assigned a female sex at birth who currently identify as female) in heterosexual relationships with cisgender partners, but research demonstrates that transgender individuals experience IPV at similar rates to heterosexual cisgender women. Despite this, there is a dearth of research examining the epidemiology of IPV among TGNC youth and the associations of both structural and intrapersonal transphobiarelated factors with IPV among this population.

Although data specific to IPV among TGNC youth are limited, some evidence demonstrates that the prevalence of IPV is especially high among TGNC populations. According to a review of the literature examining IPV among lesbian, gay, bisexual, and transgender populations, between $31.1 \%$ and $50 \%$ of TGNC individuals have reported experiencing IPV in their lifetime (Brown and Herman 2015), In addition, in the 2015 U.S. Transgender Survey conducted among a large national convenience sample of TGNC individuals, $54 \%$ of respondents reported experiencing some

Department of Health Behavior and Health Education, School of Public Health, University of Michigan, Ann Arbor, Michigan. 
form of IPV in their lifetime, including physical, emotional, and/or financial violence (James et al. 2016). Furthermore, a study by Dank and colleagues (2014) that assessed IPV among youth found that the few transgender youth in the sample $(n=18)$ reported the highest rates of IPV.

Some research has also found links between IPV and other health outcomes among TGNC populations. One study examining the relationships between HIV, IPV, victimization, substance use, and self-esteem among young transgender women in Chicago and Los Angeles found that experiences of IPV were significantly associated with polysubstance use, increased sexual risk-taking behavior, self-reports of living with $\mathrm{HIV}$, and experiences of other types of victimization (Brennan et al. 2012). Experiences of IPV among TGNC youth may be especially problematic because adolescence is a time during which dating and other forms of intimate relationships assist in the development and cultivation of skills utilized in later adult interpersonal relationships (Collins 2003; Connolly et al. 2004).

Although most research on adolescent IPV has focused on risk and protective factors at the individual and relationship levels (Vagi et al. 2013), given the complexity of TGNC youth's lives, it is important to expand the focus to structural levels of influence. Structural-level factors refer to the overarching social structure that creates cultural values (Cohen et al. 2000). At a structural level, it is important to understand how pervasive transphobic stigma contributes to the social context, in which TGNC youth experience IPV.

Transphobic stigma is a process that results in the marginalization of TGNC youth (James et al. 2016; Link and Phelan 2001). This is evident through the systematic exposure to structural vulnerabilities that limit access to resources, with great disparities in TGNC youth's experiences of poverty, homelessness, incarceration, educational attainment, employment, and sex work participation (Bauermeister et al. 2016; James et al. 2016). Limiting or controlling one's access to resources is a common component of IPV (Stark 2007), so the extant lack of resources experienced by TGNC youth may make this population especially vulnerable to IPV. For example, experiencing homelessness or housing instability may lead to reliance on intimate partners for a place to stay, and thus, they may not report IPV for fear that they would no longer have a place to stay. Pervasive societal transphobia may also contribute to IPV through increased exposure to sexual and physical violence through transphobic victimization (Garofalo et al. 2006; James et al. 2016; Kosciw et al. 2012) and the lack of safe environments where TGNC youth are free from transphobic discrimination and victimization (Grossman and D'augelli 2006).

Intrapersonal factors refer to individual characteristics, behaviors, or experiences (McLeroy et al. 1988). There are many intrapersonal factors known to co-occur with IPV among cisgender populations, including psychological distress, suicidal ideation, sexual risk-taking behaviors, and substance use (Buller et al. 2014; Coker et al. 2002). Due to experiences of stigma and minority stress (i.e., chronic psychological stress related to the experience of being stigmatized) (Meyer 1995), these co-occurring intrapersonal factors are disproportionately experienced by TGNC youth (Garofalo et al. 2006; Grossman et al. 2016; Reisner et al. 2015; Wilson et al. 2016). Therefore, it is especially important to understand how IPV among TGNC youth is related to these other psychological and behavioral health factors.
The exclusion of TGNC youth in IPV research and public health interventions contributes to a traditional gender-based heterosexual model of IPV that ignores the specific needs of this population (Brown 2007). Therefore, the purpose of the current study is to conduct an exploratory analysis, examining the associations between a range of structural and intrapersonal factors and IPV victimization among TGNC youth from 14 different cities in the United States representing various regions of the country. Gaining a better understanding of the complexity of such factors will greatly inform the development of IPV prevention and services specifically for TGNC youth experiencing this type of violence.

\section{Materials and Methods}

\section{Procedures}

Data were collected between July and December 2015 as part of the Affirming Voices for Action (AVA) project. AVA is a concurrent mixed-methods (qualitative and quantitative) study that collected data from a diverse sample of 187 TGNC youth (ages 16-24 years) in 14 cities in the United States associated with the Adolescent Medicine Trials Network for HIV/AIDS Interventions (ATN), including Baltimore, MD; Boston, MA; Chicago, IL; Denver, CO; Detroit, MI; Houston, TX; Los Angeles, CA; Memphis, TN; Miami, FL; New Orleans, LA; New York, NY; Philadelphia, PA; Tampa Bay, FL; and Washington, DC. The current analysis focuses only on the quantitative data.

Given the mixed-methods nature of the study, purposive sampling was used to recruit participants at the 14 ATN clinical sites. Staff members recruited TGNC youth from their patient population and community-based agencies serving TGNC youth. All participants recruited from the ATN clinics were living with HIV; however, communitybased strategies for recruitment targeted a range of TGNC youth living in the cities where the clinics were located. Individuals were eligible if their gender identity was not the same as the sex that they were assigned at birth; they were between the ages of 16 and 24 at the time of consent; and they were able to provide signed informed consent.

Study activities were approved by the Institutional Review Boards at all 14 sites and at the University of Michigan. Participants who were aged 18 years and older completed written informed consent and participants younger than 18 years of age provided assent, with a waiver of parental/ guardian consent due to safety concerns regarding disclosure of identity. The survey was administered using audio computer-assisted self-interview and took $\sim 45 \mathrm{~min}$. Participants were compensated for their time based on local study participation standards.

\section{Measures}

Measures in this analysis include the dependent variable (IPV) as well as structural factors, intrapersonal factors, and sociodemographic characteristics.

Intimate partner violence. IPV was measured as a dichotomous variable with the question: "Have you ever been slapped, punched, kicked, beaten up, or otherwise physically or sexually hurt by a boyfriend/girlfriend, spouse, or some other intimate partner?" Individuals who indicated 
that they did not know the answer or preferred not to answer were counted as missing $(n=11)$. This variable is limited to exclusively measuring experiences of IPV victimization and does not include psychological abuse or controlling behaviors, which have both been found to be very prevalent and harmful (Coker et al. 2000; Woodyatt et al. 2016). It is important to understand how results may differ when examining these different types of IPV; however, the measure used in this study is still useful to understand general experiences of IPV victimization.

Structural factors. Structural factors included interpersonal and individual experiences that can be proxies for structural factors, including experiences of transphobic stigma and factors that contribute to structural vulnerability (e.g., homelessness, incarceration). Systematic vulnerability refers to social conditions that increase exposure to health risks (Frohlich and Potvin 2008); these factors are important because limit access to resources, which may influence experiences of IPV (Stark 2007).

Two adapted scales were used to capture experiences of stigma: the gender-related discrimination scale and genderrelated victimization scale (Herek and Berrill 1992). The gender-related discrimination scale asks participants to indicate the number of times they experienced eight types of discrimination in the past year specifically because someone presumed the respondent to be transgender (e.g., being evicted). Since $56 \%$ of participants $(n=73)$ indicated that they did not experience any of these forms of discrimination, a dichotomous variable was used to measure discrimination.

The gender-related victimization scale asks participants to indicate the number of times they experienced 10 types of victimization in the past year specifically because someone presumed the respondent to be transgender (e.g., being spat on, being threatened, being punched, hit, kicked, or beaten). Since many participants indicated not experiencing victimization in the past year $(n=33,25.19 \%)$, but there was still a great amount of variation in responses among individuals who experienced victimization, this variable was examined as a categorical variable using a tripartite split measuring if participants experienced no victimization (0 times), some victimization (1-10 times), or a lot of victimization $(>10$ times). In addition, three aspects of structural vulnerability were included in this analysis, including lifetime experiences of incarceration, homelessness, and participation in sex work. All systematic vulnerability items were measured as dichotomous variables based on responses to yes/no questions.

Intrapersonal factors. Intrapersonal factors included depressive symptomatology, suicidal ideation, substance abuse, HIV status, and self-esteem. Depressive symptomatology was determined using the Center for Epidemiological Studies-Depression Scale, which is a 20-item scale (ranging from 0 to 60 ) used to identify depressive symptomology in the general population (Radloff 1977). Respondents who scored $\geq 16$ (a common cutoff point for this scale) (Radloff 1977) were classified as experiencing depressive symptomatology.

Substance use was measured using the CRAFFT, which is a six-item scale used to measure substance abuse and dependence among adolescents (Knight et al. 1999). The six yes or no questions in CRAFFT ask respondents to identify lifetime experiences related to the use of alcohol or drugs (e.g., Have you ever gotten into trouble while you were using alcohol or drugs?) (Knight et al. 1999). Respondents who answered yes to at least two of the items were classified as experiencing substance abuse (Knight et al. 2002).

HIV status was measured as a dichotomous variable based on whether or not a respondent reported a known positive HIV serostatus. Respondents who had never tested positive for HIV (including individuals who did not know their status) were classified as not having a known positive HIV status. Lifetime suicidal ideation was also measured as a dichotomous variable based on a yes or no question, and, finally, self-esteem was determined based on a single validated question (Robins et al. 2001): "I feel that I'm a person of worth, at least on an equal plane with others." Responses ranged on a scale from 1 (definitely agree) to 5 (definitely disagree).

Sociodemographic characteristics. Demographic factors included age, race, gender identity, education, and United States region. Age was measured in years as a continuous variable, and race was measured using three categories (nonHispanic white, non-Hispanic black, or another race); white was the reference group. Gender identity was measured as a categorical variable, including transfeminine, transmasculine, and nonconforming gender identities. Transfeminine was defined as participants who reported being assigned a male sex at birth, who currently identify as a woman or a transgender woman; transmasculine was defined as participants who reported being assigned a female sex at birth, who currently identify as a man or as a transgender man; and gender nonconforming included participants who identified as gender queer, gender nonconforming, or another gender. Education was measured as a dichotomous variable $(0=$ did not graduate high school and not currently in school; $1=$ graduated from high school or currently in school). United States region was measured as a five-category variable and included the following: Northeast, Mid-Atlantic, Midwest, West, and South; Northeast was the reference group.

\section{Data analysis}

We fit two separate logistic regression models to examine structural and intrapersonal factors associated with the occurrence of IPV. Model 1 included all structural variables as predictors and Model 2 included all intrapersonal variables as predictors. Both models also included sociodemographic variables as covariates. All participants with missing data from either model $(n=55)$ were not included in the analysis. To ensure that there was no systematic bias resulting from missing data, we used chi-square and $t$-tests to determine that there were no meaningful differences in the demographics (age, race, gender identity, education) between respondents with and without missing data. An alpha level of 0.05 was used to determine statistical significance.

\section{Results}

A total of 131 participants were included in the analysis. Descriptive statistics are demonstrated in Table 1. The mean age of the sample was 20.9 (standard deviation $=2.19$ ), with nearly half of the participants identifying as non-Hispanic black and 75\% identifying as transfeminine. Reports of IPV 
Table 1. Descriptive Statistics $(N=131)$

\begin{tabular}{|c|c|c|c|}
\hline Variables & $\begin{array}{c}I P V \% \\
(\mathrm{n})\end{array}$ & $\begin{array}{c}\text { No IPV } \% \\
\text { (n) }\end{array}$ & $\begin{array}{l}\text { Total \% } \\
\text { (n) }\end{array}$ \\
\hline Any discrimination & $51.72(30)$ & 54.96 & $44.27(58)$ \\
\hline \multicolumn{4}{|l|}{ Victimization } \\
\hline None & $21.21(7)$ & $78.79(26)$ & $25.19(33)$ \\
\hline $1-10$ times & $36.17(17)$ & $63.83(30)$ & $35.88(47)$ \\
\hline$>10$ times & $45.04(59)$ & $54.96(72)$ & $38.93(51)$ \\
\hline Sex work & $62.71(37)$ & $37.29(22)$ & $45.04(59)$ \\
\hline Ever incarcerated & $66.00(33)$ & $34.00(17)$ & $38.17(50)$ \\
\hline Ever homeless & $59.02(36)$ & $40.98(25)$ & $46.56(61)$ \\
\hline $\begin{array}{l}\text { Depressive } \\
\text { symptomatology }\end{array}$ & $64.94(50)$ & $35.06(27)$ & $58.78(77)$ \\
\hline Suicide ideation & $52.70(39)$ & $47.30(35)$ & $56.49(74)$ \\
\hline Substance abuse & $52.87(46)$ & $47.13(41)$ & $66.41(87)$ \\
\hline $\begin{array}{l}\text { Self-esteem, } \\
\text { mean (SD) }\end{array}$ & $1.97(1.08)$ & $1.60(1.00)$ & $1.76(1.05)$ \\
\hline HIV status & $55.26(21)$ & $44.74(17)$ & $29.01(38)$ \\
\hline $\begin{array}{l}\text { Graduated from } \\
\text { or currently } \\
\text { in high school }\end{array}$ & $43.86(50)$ & $56.14(64)$ & $87.02(114)$ \\
\hline \multicolumn{4}{|l|}{ Race } \\
\hline Non-Hispanic white & $47.62(10)$ & $52.38(11)$ & $16.03(21)$ \\
\hline Non-Hispanic black & $45.00(27)$ & $55.00(33)$ & $45.80(60)$ \\
\hline Other race & $44.00(22)$ & $56.00(28)$ & $38.17(50)$ \\
\hline \multicolumn{4}{|l|}{ Gender identity } \\
\hline Transfeminine & $43.59(34)$ & $56.41(44)$ & $59.54(78)$ \\
\hline Transmasculine & $30.43(7)$ & $69.57(16)$ & $17.56(23)$ \\
\hline $\begin{array}{l}\text { Gender } \\
\text { nonconforming }\end{array}$ & $60.00(18)$ & $40.00(12)$ & $22.90(30)$ \\
\hline \multicolumn{4}{|l|}{ United States region } \\
\hline Northeast & $38.10(8)$ & $61.90(13)$ & $16.03(21)$ \\
\hline Mid-Atlantic & 25.53 (4) & $76.47(13)$ & $12.98(17)$ \\
\hline Midwest & $48.15(13)$ & $51.85(14)$ & $20.61(27)$ \\
\hline West & $45.45(10)$ & $54.55(12)$ & $16.79(22)$ \\
\hline South & $54.55(24)$ & $45.45(20)$ & $33.59(44)$ \\
\hline Age, mean (SD) & $21.07(1.97)$ & $20.47(2.33)$ & $20.74(2.19)$ \\
\hline Total & $45.04(59)$ & $54.96(72)$ & 131 \\
\hline
\end{tabular}

$\mathrm{IPV}$, intimate partner violence; SD, standard deviation.

were high, with $45 \%$ reporting lifetime experiences of physical or sexual IPV. Nearly half of the participants experienced gender-related discrimination in the past year, with experiences of victimization being more common (75\%). Approximately $45 \%$ of participants had ever participated in sex work, $38 \%$ had ever been incarcerated, and $47 \%$ had experienced homelessness at some point in their lives. Reports of poor health outcomes were also high, with $29 \%$ reporting living with HIV, nearly $60 \%$ reporting depressive symptomatology and suicide ideation, and approximately two-thirds reporting substance abuse.

For Model 1, the structural variables that were associated with IPV were experiencing $>10$ incidents of transphobic victimization in the past year, participation in sex work, and a history of incarceration (Table 2). Compared with participants who experienced no victimization, those who experienced $>10$ incidents were 6.37 times more likely to report experiencing IPV $(p<0.001)$. Participants who engaged in sex work were 4.52 times more likely to report IPV $(p=0.008)$ and those who reported a history of incarceration were 3.63 times more likely to report IPV $(p=0.041)$. None of the other variables were statistically significant in this model. For Model 2, the only intrapersonal variable that was associated with IPV was depressive symptomatology (Table 3). However, the association between depressive symptomatology and IPV was strong, with participants being 7.65 times more likely to report IPV if they experienced symptoms of depression $(p<0.001)$.

\section{Discussion}

Among our sample, $45 \%$ reported a lifetime history of IPV. This is consistent with previous literature examining IPV among TGNC populations (Brown and Herman 2015; James et al. 2016). In addition, as with other studies of TGNC youth (Bauermeister et al. 2016; Garofalo et al. 2006), this study found high reports of gender-related discrimination and victimization, environmental and structural stressors (including participation in sex work, incarceration, and homelessness), and high reports of HIV, depressive symptomatology, and substance abuse. Although $29 \%$ of our sample reported living with HIV, we purposively sampled for youth who were living with HIV given the nature of the AVA study.

In our structural-level logistic regression model, we found that youth who had experienced extreme victimization, participated in sex work, or had a history of incarceration were much more likely to have experienced IPV. In our intrapersonal-level regression model, only one factor was significant, demonstrating that those who reported symptoms of depression were 7.65 times more likely to report a history of IPV.

Unlike previous studies conducted among TGNC youth (Brennan et al. 2012), substance use and HIV status were not significantly associated with IPV; this difference may be attributed to the definition of IPV used in this study or the national sample of TGNC youth from 14 cities in the United States. In addition, since this was a cross-sectional sample of youth, we cannot infer directionality or causality of these relationships. For example, it may be that those youth who are experiencing major depressive symptoms are more likely to experience IPV, or it may be that experiencing IPV can lead to symptoms of major depression. Still, these significant relationships demonstrate the importance of understanding the connections between TGNC youth's social context and experiences of IPV.

These relationships also highlight the multiple ways in which transphobic stigma may influence TGNC youth's experiences, well-being, and relationships. Transphobia simultaneously normalizes cisgender identity while labeling TGNC individuals as an "othered" group; this process results in status loss, which can occur through limited access to resources and systematic vulnerability (Link and Phelan 2001). At the same time, this process of stigmatization limits the power and control that TGNC youth have when navigating societal institutions (e.g., education, housing) (Graham 2014) and may also limit the agency they are able to attain within intimate relationships. To adequately address IPV among TGNC youth, it is important to address transphobic stigma and the multiple ways in which it contributes to poor health (Hatzenbuehler et al. 2013). 


\begin{tabular}{|c|c|c|c|}
\hline & OR & $95 \% C I$ & p-Value \\
\hline Any discrimination & 0.95 & $0.38,2.41$ & 0.920 \\
\hline \multicolumn{4}{|l|}{ Victimization } \\
\hline None & Reference group & & \\
\hline $1-10$ times & 3.06 & $0.90,10.43$ & 0.074 \\
\hline$>10$ times & 6.37 & $1.85,21.93$ & $0.003 *$ \\
\hline Sex work & 4.52 & $1.62,12.56$ & $0.004 *$ \\
\hline Ever incarcerated & 3.63 & $1.26,10.44$ & $0.017 * *$ \\
\hline Ever homeless & 1.55 & $0.57,4.19$ & 0.386 \\
\hline Age & 0.99 & $0.79,1.24$ & 0.928 \\
\hline $\begin{array}{l}\text { Graduated from } \\
\text { or currently } \\
\text { in high school }\end{array}$ & 0.64 & $0.16,2.54$ & 0.527 \\
\hline \multicolumn{4}{|l|}{ Race } \\
\hline Non-Hispanic white & Reference group & & \\
\hline Non-Hispanic black & 0.59 & $0.14,2.45$ & 0.467 \\
\hline Other & 0.48 & $0.13,1.83$ & 0.285 \\
\hline \multicolumn{4}{|l|}{ Gender identity } \\
\hline Transfeminine & Reference group & & \\
\hline Transmasculine & 1.23 & $0.32,4.70$ & 0.758 \\
\hline $\begin{array}{l}\text { Gender } \\
\text { nonconforming }\end{array}$ & 2.42 & $0.81,7.30$ & 0.115 \\
\hline \multicolumn{4}{|l|}{ United States region } \\
\hline Northeast & Reference group & & \\
\hline Mid-Atlantic & 0.23 & $0.03,1.53$ & 0.128 \\
\hline Midwest & 0.92 & $0.19,4.42$ & 0.922 \\
\hline West & 1.26 & $0.29,5.44$ & 0.757 \\
\hline South & 1.83 & $0.46,7.38$ & 0.393 \\
\hline
\end{tabular}

$* p<0.01 ; * * p<0.05$.

OR, odds ratio; $\mathrm{CI}$, confidence interval.

\section{Screening and providing IPV services for TGNC youth}

Given the high rates of IPV reported by this sample of TGNC youth, along with IPV's associations with sex work, incarceration, victimization, and depressive symptomatology, it is important to increase health and social service providers' awareness of the need to screen for IPV among TGNC youth. Given the strong association between IPV and depressive symptoms, it will be especially prudent for mental healthcare providers to assess for IPV among their TGNC clients.

In order for providers to effectively screen for IPV, culturally and developmentally appropriate instruments need to be developed and validated for TGNC youth. Previous research demonstrates that different marginalized populations have unique experiences of IPV and therefore require distinct screening tools (Stephenson and Finneran 2013). Furthermore, TGNC youth may be reluctant to report IPV if they fear that this will cause additional barriers for receiving gender affirmation services.

Once IPV is identified, TGNC youth need trans-informed and culturally aware providers. Currently, TGNC people receive unequal treatment in domestic violence programs, especially if they are low-income, not United States citizens, have disabilities, or are more frequently perceived to be transgender (Goodmark 2013; Seelman 2015). TGNC youth deserve IPV-related services that are not only free of discrimination but also consider their unique lived experiences and the complexity of their relationships.
Table 3. Logistic Regression Results FOR INTIMATE PARTNER Violence AND INTRAPERSONAL VARIABLES $(N=131)$

\begin{tabular}{lccc}
\hline & OR & $95 \%$ CI & p-Value \\
\hline $\begin{array}{l}\text { Depressive } \\
\text { symptomatology }\end{array}$ & 7.65 & $2.94,19.92$ & $<0.001^{*}$ \\
Suicide ideation & 1.64 & $0.65,4.18$ & 0.296 \\
Substance abuse & 2.28 & $0.87,5.97$ & 0.093 \\
Self esteem & 1.11 & $0.72,1.69$ & 0.640 \\
HIV status & 1.69 & $0.58,4.97$ & 0.340 \\
Age & 1.13 & $0.92,1.39$ & 0.233 \\
Graduated from & 0.91 & $0.26,3.15$ & 0.886 \\
$\quad$ or currently & & & \\
in high school & & & \\
Race & & & \\
$\quad$ Non-Hispanic white & Reference group & & \\
Non-Hispanic black & 1.18 & $0.30,4.65$ & 0.814 \\
$\quad$ Other & 1.24 & $0.34,4.58$ & 0.747 \\
Gender identity & & & \\
$\quad$ Transfeminine & Reference group & & \\
$\quad$ Transmasculine & 0.50 & $0.14,1.74$ & 0.276 \\
$\quad$ Gender & 1.29 & $0.45,3.69$ & 0.638 \\
$\quad$ nonconforming & & & \\
United States region & & & \\
$\quad$ Northeast & Reference group & & \\
Mid-Atlantic & 0.51 & $0.08,3.16$ & 0.466 \\
Midwest & 1.34 & $0.31,5.74$ & 0.694 \\
West & 0.87 & $0.20,3.76$ & 0.854 \\
South & 1.44 & $0.38,5.49$ & 0.593 \\
\hline
\end{tabular}

${ }^{*} p<0.001$.

Prevention efforts are also needed and should be delivered in the various settings and sectors that have contact with TGNC youth. These programs need to be sensitive to the structurallevel factors that may perpetuate the development and maintenance of IPV. Given our findings, it would be beneficial to provide prevention (and treatment) services to TGNC youth in community-based organizations and clinical settings that provide services to TGNC youth and facilities addressing juvenile justice, psychiatric care, and homelessness.

\section{Limitations, strengths, and future research}

There were some limitations in this study. Since this was a cross-sectional sample, causal inferences cannot be made. In addition, caution should be taken before generalizing results to TGNC youth outside of the ATN catchment areas included in this study. This study used convenience sampling, which limits generalizability; however, this is a common recruitment method for hard-to-reach populations (Muhib et al. 2001). The sample size of 131 is also fairly small for conducting logistic regression, making it difficult to detect significant relationships; however, since significant relationships were detected even with a small sample size, this suggests robust findings. In addition, even though the sample size is small for a general population, a sample size of 131 in this study is uniquely large given the focus demographic of transgender youth. A larger sample size would allow for more complicated analysis and would enable the inclusion of additional variables and the examination of variables across the socioecological model to all be included in the same model. To generally 
assess IPV, this study focused specifically on sexual and physical IPV, but future research should consider more nuanced experiences of IPV, such as emotional IPV and controlling behaviors. This measure is also based on self-report, which does not capture experiences of IPV among individuals who fear reporting or are unable to identify IPV. Despite these limitations, these findings can help elucidate the social, behavioral, and health factors that are associated experiences of IPV among TGNC youth.

Future research should further explore multiple ecological factors that influence IPV among TGNC youth. This may include a focus on resilience, as many TGNC youth navigate intimate relationships without any reported violence. Given the importance of identity exploration and formation during adolescence and early adulthood, future research will also be enhanced by applying an intersectional framework (Harper and Wilson 2017) to the study of IPV among TGNC youth through an increased understanding of the influence of multiple identities that all partners bring to the intimate interaction and how these multiple identities are tied to experiences of power and control within the relationship.

Finally, future explorations of IPV among TGNC youth should be sensitive to assumptions regarding the composition of romantic relationships that involve one or more TGNC individuals; as TGNC youth may engage in romantic relationships with individuals or groups that display a range of sexual orientations and/or gender identities and expressions. Research and programs will also benefit from exploring the potential influence of both TGNC youth's and researchers'/providers' relationship heteronormativity biases, which refers to a belief that heterosexuality and heterosexual relationships are the societal norm (Herek and Garnets 2007). TGNC youth may internalize a heteronormative bias and thus may view their relationships as non-normative or even deviant; which may impact their ability to identify hurtful dynamics and their willingness to seek services (Blasko et al. 2007; Herek and Garnets 2007).

\section{Acknowledgments}

This work was supported by The Adolescent Medicine Trials Network for HIV/AIDS Interventions (ATN) from the National Institutes of Health [U01 HD 040533 and U01 HD 040474] through the National Institute of Child Health and Human Development (B. Kapogiannis, S. Lee), with supplemental funding from the National Institutes on Drug Abuse and Mental Health. The views and opinions expressed in this report are those of the authors and should not be construed to represent the views of any of the sponsoring organizations, agencies, or the U.S. Government. We would also like to thank the following individuals, groups, and entities for their contributions to this study: Elliot Popoff and Bré Anne Campbell, University of Michigan research staff who made this study possible. The investigators and staff at the following sites that participated in this study: University of South Florida, Tampa (Emmanuel, Straub, Bruce, Kerr), Children's Hospital of Los Angeles (Belzer, Tucker, Franco), Children's National Medical Center (D’Angelo, Trexler, Carr, Sinkfield), Children's Hospital of Philadelphia (Douglas, Tanney, DiBenedetto, Franklin, Smith), John H. Stroger Jr. Hospital of Cook County and the Ruth M. Rothstein CORE Center (Henry-Reid, Bojan, Balthazar), Montefiore Medical Center (Futterman, Campos,
Wesp, Nazario, Reopell), Tulane University Health Sciences Center (Abdalian, Kozina, Baker, Wilkes), University of Miami School of Medicine (Friedman, Maturo), St. Jude's Children's Research Hospital (Gaur, Flynn, Dillard, HurdSawyer), Baylor College of Medicine (Paul, Head, Sierra), Wayne State University (Secord, Cromer, Walters, Houston), Johns Hopkins University (George-Agwu, Anderson, Worrel-Thorne), Fenway Institute (Mayer, Dormitzer, Massaquoi, Gelman), University of Colorado Denver (Reirden, Hahn, Bernath). Network, scientific and logistical support was provided by the ATN Coordinating Center (C. Wilson, C. Partlow) at The University of Alabama at Birmingham and the ATN 130 protocol team. Network operations and data management support was provided by the ATN Data and Operations Center at Westat, Inc. (G. Price). The authors are grateful to the members of the Affirming Voices for Action (AVA) Youth Advisory Board for their insight and guidance. We would like to thank the trans youth who raised their voices and shared their experiences with us. We hear you.

\section{Author Disclosure Statement}

No competing financial interests exist.

\section{References}

Bauermeister JA, Goldenberg T, Connochie D, et al. (2016). Psychosocial disparities among racial/ethnic minority transgender young adults and young men who have sex with men living in Detroit. Transgend Health. 1, 279-290.

Blasko KA, Winek JL, Bieschke KJ. (2007). Therapists' prototypical assessment of domestic violence situations. J Marital Fam Ther. 33, 258-269.

Brennan J, Kuhns LM, Johnson AK, et al. (2012). Syndemic theory and HIV-related risk among young transgender women: The role of multiple, co-occurring health problems and social marginalization. Am J Public Health. 102, 1751-1757.

Brown N. (2007). Stories from outside the frame: Intimate partner abuse in sexual-minority women's relationships with transsexual men. Femin Psychol. 17, 373-393.

Brown TN, Herman JL. (2015). Intimate Partner Violence and Sexual Abuse Among LGBT People. Los Angeles, CA: The Williams Institute.

Buller AM, Devries KM, Howard LM, et al. (2014). Associations between intimate partner violence and health among men who have sex with men: A systematic review and meta-analysis. PLoS Med. 11, e1001609.

Cohen DA, Scribner RA, Farley TA. (2000). A structural model of health behavior: A pragmatic approach to explain and influence health behaviors at the population level. Prev Med. 30, 146-154.

Coker AL, Davis KE, Arias I, et al. (2002). Physical and mental health effects of intimate partner violence for men and women. Am J Prev Med. 23, 260-268.

Coker AL, Smith PH, Bethea L, et al. (2000). Physical health consequences of physical and psychological intimate partner violence. Arch Fam Med. 9, 451.

Collins WA. (2003). More than myth: The developmental significance of romantic relationships during adolescence. J Res Adolesc. 13, 1-24.

Connolly J, Craig W, Goldberg A, et al. (2004). Mixed-gender groups, dating, and romantic relationships in early adolescence. J Res Adolesc. 14, 185-207.

Dank M, Lachman P, Zweig JM, et al. (2014). Dating violence experiences of lesbian, gay, bisexual, and transgender youth. J Youth Adolesc. 43, 846-857. 
Frohlich KL, Potvin L. (2008). Transcending the known in public health practice: The inequality paradox: The population approach and vulnerable populations. Am J Public Health. 98, 216-221.

Garofalo R, Deleon J, Osmer E, et al. (2006). Overlooked, misunderstood and at-risk: Exploring the lives and HIV risk of ethnic minority male-to-female transgender youth. J Adolesc Health. 38, 230-236.

Goodmark L. (2013). Transgender people, intimate partner abuse, and the legal system. Harv CR-CLL Rev. 48, 51.

Graham LF. (2014). Navigating community institutions: Black transgender women's experiences in schools, the criminal justice system, and churches. Sex Res Soc Policy. 11, 274-287.

Grossman AH, D'augelli AR. (2006). Transgender youth: Invisible and vulnerable. J Homosex. 51, 111-128.

Grossman AH, Park JY, Russell ST. (2016). Transgender youth and suicidal behaviors: Applying the interpersonal psychological theory of suicide. J Gay Lesbian Mental Health. 20, 329-349.

Harper GW, Wilson BD. (2017). Situating sexual orientation and gender identity diversity in context and communities. In APA Handbook of Community Psychology: Theoretical Foundations, Core Concepts, and Emerging Challenges. M.A. Bond, I. SerranoGarcia, C.B. Keys, M. Shinn, eds. (American Psychological Association, Washington, DC), Vol. 1, pp. 387-402.

Hatzenbuehler ML, Phelan JC, Link BG. (2013). Stigma as a fundamental cause of population health inequalities. Am J Public Health. 103, 813-821.

Herek GM, Berrill KT. (1992). Hate Crimes: Confronting Violence Against Lesbians and Gay Men. Newbury Park, CA: Sage.

Herek GM, Garnets LD. (2007). Sexual orientation and mental health. Annu Rev Clin Psychol. 3, 353-375.

James SE, Herman JL, Rankin S, et al. (2016). The Report of the 2015 U.S. Transgender Survey. Retrieved from Washington, DC. http:// www.ustranssurvey.org/

Knight JR, Sherritt L, Shrier LA, et al. (2002). Validity of the CRAFFT substance abuse screening test among adolescent clinic patients. Arch Pediatr Adolesc Med. 156, 607-614.

Knight JR, Shrier LA, Bravender TD, et al. (1999). A new brief screen for adolescent substance abuse. Arch Pediatr Adolesc Med. 153, 591-596.

Kosciw JG, Greytak EA, Bartkiewicz MJ, et al. (2012). The 2011 National School Climate Survey: The Experiences of Lesbian, Gay, Bisexual and Transgender Youth in Our Nation's Schools. New York, NY: GLSEN.

Langenderfer-Magruder L, Whitfield DL, Walls NE, et al. (2016). Experiences of intimate partner violence and subsequent police reporting among lesbian, gay, bisexual, transgender, and queer adults in Colorado: Comparing rates of cisgender and transgender victimization. J Interpers Violence. 31, 855-871.

Link BG, Phelan JC. (2001). Conceptualizing stigma. Annu Rev Sociol. 363-385.
Lombardi EL, Wilchins RA, Priesing D, et al. (2002). Gender violence: Transgender experiences with violence and discrimination. J Homosex. 42, 89-101.

McLeroy KR, Bibeau D, Steckler A, et al. (1988). An ecological perspective on health promotion programs. Health Educ Behav. 15, 351-377.

Meyer IH. (1995). Minority stress and mental health in gay men. J Health Soc Behav. 36, 38-56.

Muhib F, Lin L, Stueve A, et al. (2001). A venue-based method for sampling hard-to-reach populations. Public Health Rep. 116, 216-222.

Radloff LS. (1977). The CES-D scale: A self-report depression scale for research in the general population. Appl Psychol Meas. 1, 385-401.

Reisner SL, Greytak EA, Parsons JT, et al. (2015). Gender minority social stress in adolescence: Disparities in adolescent bullying and substance use by gender identity. J Sex Res. 52, 243-256.

Robins RW, Hendin HM, Trzesniewski KH. (2001). Measuring global self-esteem: Construct validation of a single-item measure and the Rosenberg Self-Esteem Scale. Pers Soc Psychol Bull. 27, 151-161.

Seelman KL. (2015). Unequal treatment of transgender individuals in domestic violence and rape crisis programs. J Soc Serv Res. 41, $307-325$.

Stark E. (2007). Coercive Control: How Men Entrap Women in Personal Life. (Oxford University Press, New York, NY).

Stephenson R, Finneran C. (2013). The IPV-GBM scale: A new scale to measure intimate partner violence among gay and bisexual men. PLoS One. 8, e62592.

Stotzer RL. (2009). Violence against transgender people: A review of United States data. Aggress Violent Behav. 14, 170-179.

Vagi KJ, Rothman EF, Latzman NE, et al. (2013). Beyond correlates: A review of risk and protective factors for adolescent dating violence perpetration. J Youth Adolesc. 42, 633-649.

Wilson EC, Chen Y-H, Arayasirikul S, et al. (2016). The impact of discrimination on the mental health of trans* female youth and the protective effect of parental support. AIDS Behavior. 20, 2203-2211.

Woodyatt CR, Finneran CA, Stephenson R. (2016). In-person versus online focus group discussions: A comparative analysis of data quality. Qual Health Res. 26, 741-749.

World Health Organization. (2010). Preventing Intimate Partner and Sexual Violence Against Women: Taking Action and Generating Evidence. (World Health Organiztion, Geneva, Switzerland).

Address correspondence to:

Tamar Goldenberg, $\mathrm{MPH}$ University of Michigan

Center for Sexuality and Health Disparities

400 North Ingalls Building Ann Arbor, MI 48109

E-mail: tamargol@umich.edu 